\title{
In vitro antineoplastic effects of brivaracetam and lacosamide on human glioma cells
}

\author{
Ambra Rizzo ${ }^{1+}$, Sara Donzelli ${ }^{2+}$, Vita Girgenti ${ }^{1}$, Andrea Sacconi ${ }^{2}$, Chiara Vasco ${ }^{1}$, Andrea Salmaggi $^{3}$, \\ Giovanni Blandino ${ }^{2}$, Marta Maschio ${ }^{4^{*}}$ and Emilio Ciusani ${ }^{1}$
}

\begin{abstract}
Background: Epilepsy is a frequent symptom in patients with glioma. Although treatment with antiepileptic drugs is generally effective in controlling seizures, drug-resistant patients are not uncommon. Multidrug resistance proteins (MRPs) and P-gp are over-represented in brain tissue of patients with drug-resistant epilepsy, suggesting their involvement in the clearance of antiepileptic medications. In addition to their anticonvulsant action, some drugs have been documented for cytotoxic effects. Aim of this study was to evaluate possible in vitro cytotoxic effects of two new-generation antiepileptic drugs on a human glioma cell line U87MG.
\end{abstract}

Methods: Cytotoxicity of brivaracetam and lacosamide was tested on U87MG, SW1783 and T98G by MTS assay. Expression of chemoresistance molecules was evaluated using flow cytometry in U87MG and human umbilical vein endothelial cells (HUVECs). To investigate the putative anti-proliferative effect, apoptosis assay, microRNA expression profile and study of cell cycle were performed.

Results: Brivaracetam and lacosamide showed a dose-dependent cytotoxic and anti-migratory effects. Cytotoxicity was not related to apoptosis. The exposure of glioma cells to brivaracetam and lacosamide resulted in the modulation of several microRNAs; particularly, the effect of miR-195-5p modulation seemed to affect cell cycle, while miR-107 seemed to be implicated in the inhibition of cells migration. Moreover, brivaracetam and lacosamide treatment did not modulate the expression of chemoresistance-related molecules MRPs1-3-5, GSTR, P-gp on U87MG and HUVECS.

Conclusion: Based on antineoplastic effect of brivaracetam and lacosamide on glioma cells, we assume that patients with glioma could benefit by the treatment with these two molecules, in addition to standard therapeutic options.

Keywords: Lacosamide, Brivaracetam, Brain tumor-related epilepsy, Cytotoxicity, Glioma

\section{Background}

Brain tumors are among the most disabling and resource-consuming diseases in neurology [1-4]. A common clinical manifestation of brain tumors is brain tumor-related epilepsy; in younger patients, with a long life expectancy, epilepsy is often the major problem, whereas in patients with high grade gliomas, seizures are

\footnotetext{
* Correspondence: marta.maschio@ifo.gov.it

${ }^{\dagger}$ Equal contributors

${ }^{4}$ Center for tumor-related epilepsy, Area of Supporting Care, Regina Elena National Cancer Institute, Via Elio Chianesi 53, 00144 Rome, Italy

Full list of author information is available at the end of the article
}

part of a more complex scenario involving cognitive deterioration and motor/sensory deficits [5-7].

The management of brain tumor-related epilepsy must keep in consideration issues pertaining to both epileptology and neuro-oncology. Side effects of anti-epileptic drugs (AED) have in fact a relevant role; indeed, enzyme-inducing anticonvulsant drugs (EIAED) may interfere with the metabolism of chemotherapeutic agents $[8,9]$. Moreover, antiepileptic drugs per se may display an activity on brain cancer cells, as suggested by in vitro experiments [10].

In 2009 Jaeckle had analysed prospectively the EIAED use in correlation with the outcome in patients with 
newly diagnosed glioblastoma (GBM). They found that overall survival (OS) and progression-free survival (PFS) were significantly longer in patients receiving EIAED compared to patients receiving non-EIAED [11].

On the other hand, in 2011 Weller et al. examined the impact of the interaction between AED use and chemoradiotherapy on survival in patients with newly diagnosed GBM. The OS of patients who were receiving AED at baseline versus not-receiving any AED was similar. However, patients receiving valproic acid (VPA) alone appeared to derive more survival benefit from chemo-radiotherapy (HR 0.39, 95\% CI: 0.24-0.63) than patients receiving EIAED (HR 0.69, 95\% CI: 0.53-0.90) or patients not receiving any AED (HR 0.67, 95\% CI: 0.49-0.93) [12].

In this context, particular attention has been devoted to two non-EIAED: VPA, known for a long time as a histone deacetylase inhibitor [13] and levetiracetam (LEV).

In 2013, Guthrie investigated the role of VPA as an antitumor agent in the management of patients with GBM. The results showed that patients treated with AED had a significantly longer survival than those who were not. Moreover, patients receiving VPA had a significantly longer survival than both those who did not receive AED and those who received other AED [14].

Kerkhof studied the effect of VPA on survival of patients with newly diagnosed GBM. The group using VPA and temozolomide (TMZ) during at least 3 months had a significantly longer median survival compared with what observed in the group not using VPA or using another AED (69 vs 61 weeks) [15].

As far as LEV is concerned, in 2010 Bobustuc hypothesized that AED may modulate O-6 methylguanineDNA methyltransferase (MGMT), a DNA repair protein that has an important role in tumor cell resistance to alkylating agents, and LEV was reported as the most potent MGMT inhibitor among several AED [10].

Recently, Kim analysed the benefit of LEV compared with other AED as a chemosensitizer to TMZ for patients with GBM [16]. The median PFS and OS for patients who received LEV in combination with TMZ were significantly longer than those for patients who did not receive LEV (6.7 vs 9.4 months and 16.7 vs 25.7 months respectively).

The possible actions of antiepileptic drugs on brain cancer cells include activity on cell proliferation, apoptosis and migration [17]. A number of intracellular pathways are involved in these activities, among which microRNA (miRNA) are gaining increasing attention. Many data suggest that miRNA are key components of a wide range of biological processes [18-20] and in a recently published study, in which miRNAs expression profiling was analyzed in a cohort of patients affected by low grade-gliomas, miR-196b has been identified as a predictive marker of seizures occurrence [21].

Among drugs recently introduced in the management of epilepsy, both lacosamide (LCM) and brivaracetam (BRV) are devoid of enzyme-inducing activity on the cytochrome system, being good candidates for introduction in the management of brain tumor epilepsy. Lacosamide has an inhibitory activity on histone deacetylase [22], making it worthwhile investigating its in vitro effects on brain cancer, while brivaracetam, a parent compound of levetiracetam, might share with it the same biological effects and both have been recently described to have high brain permeability [23, 24].

In the present study, we investigated in vitro on a human glioma cell line, the effects of brivaracetam and lacosamide on biological parameters involved in tumor growth, resistance to therapies and invasiveness.

\section{Methods}

Cell cultures

The human glioma cell lines U87MG, SW1483 and T98G and human fibroblasts were purchased from ATCC (LGC Standards S.r.l.). Cells were cultured in Dulbecco's Modified Eagle's Medium (DMEM, Gibco) supplemented with $10 \%$ foetal bovine serum (FBS, Gibco) and 1\% penicillin/streptomycin (Gibco).

Human umbilical vein endothelial cells (HUVECs) primary cultures were isolated from healthy donors [25] and used in experiments up to the tenth passage. Purity of cell cultures was higher than $95 \%$ as assessed by flow cytometry after CD31 staining (polyclonal phycoerythrinconjugated goat anti-human CD31, BD Bioscience). HUVECs were cultured in complete Endothelial Basal Medium (Lonza) supplemented with growth factors and antibiotics (EGM Single Quots, Lonza).

\section{Drugs}

BRV and LCM were kindly provided by UCB Pharma. Drugs were dissolved in distilled water at a concentration of $100 \mathrm{mM}$ for BRV and $50 \mathrm{mM}$ for LCM and successively diluted in complete medium to the necessary experimental concentrations (BRV: 100-200-400-600-8001000-1200-1800-2000 $\mu \mathrm{M}$; LCM: 100-200-400-600800-1000-1200-1600-2000-2400 $\mu \mathrm{M})$.

\section{Cytotoxicity}

Cytotoxicity of BRV and LCM was studied by cell proliferation assay following the manufacturer's protocol (MTS assay, Molecular Probes).

Inhibitory concentrations (IC) were calculated from the regression line that associates percentage of inhibition and drug concentrations as follows: $100-(100 \mathrm{x}$ average n.cells $\mathrm{x}$ $\mathrm{C} / \mathrm{n}$. cell basal level). Where $\mathrm{C}=$ drug concentration [range $0-2500 \mu \mathrm{M}$ ]. 


\section{Apoptosis}

Evaluation of apoptotic cells was performed in treated cells (IC20 BRV or IC20 LCM at 24-48-72 h) and in untreated cells (control) using Annexin $\mathrm{V}$ binding assay (Immunostep) for flow citometry (FacsVantage SE, Becton Dickinson) following the manufacturer's protocol.

\section{Chemoresistance}

Treated and untreated cells were fixed and permeabilized using Cytofix/CytopermTM Fixation/Permeabilization Kit (BD Biosciences) for the evaluation of the expression of chemoresistance-associated molecules. Cells were then stained with the following antibodies: mouse-antihuman

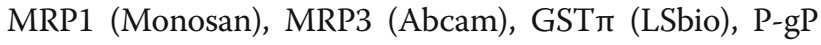
(Chemicon) and rat-anti-human MRP5 (Kamiya) primary antibodies for $1 \mathrm{~h}$ at $4{ }^{\circ} \mathrm{C}$ and subsequently $30 \mathrm{~min}$ at $4{ }^{\circ} \mathrm{C}$ with a secondary conjugated antibody for flow cytometry: goat-anti-mouse fluorescein isothiocyanate-conjugated antibody (BD Biosciences) for MRP1, MRP3, GST $\pi$ and $\mathrm{P}-\mathrm{gP}$ and goat anti rat-biotin streptavidin phycoerythrin conjugated antibody for MRP5. Cells were analyzed by flow-cytometry.

\section{Cell cycle}

Cells $\left(8 \cdot 10^{\wedge} 5\right)$ were plated in triplicate and cultured for 24 h. LCM $300 \mu \mathrm{M}$ (IC20) and BRV $400 \mu \mathrm{M}$ (IC20) were then added for $72 \mathrm{~h}$. At each time-point cells were harvested and fixed in ethanol $80 \%$ at $4{ }^{\circ} \mathrm{C}$ for $30 \mathrm{~min}$, washed and stained with PI $50 \mu \mathrm{g} / \mathrm{ml}$ in PBS overnight at $4{ }^{\circ} \mathrm{C}$. DNA content was evaluated using flowcytometry.

\section{MiRNA expression}

Total RNA was extracted using the TRIZOL (Gibco). The concentration and purity of total RNA were assessed using a Nanodrop TM 1000 (Nanodrop Technologies). Total RNA (100 ng) was labelled and hybridized to Human miRNA Microarray V.19 (Agilent). Scanning and image analysis were performed using the Agilent DNA Microarray Scanner (P/N G2565BA) equipped with extended dynamic range (XDR) software according to the Agilent miRNA Microarray System with miRNA Complete Labeling and Hyb Kit protocol manual. Feature Extraction Software (Version 10.5) was used for data extraction from raw microarray image files using the miRNA_105_Dec08 FE protocol.

\section{Formaldehyde cross-linking and chromatin immunoprecipitation}

Formaldehyde cross-linking and chromatin immunoprecipitations were performed as previously described. The chromatin solution was immunoprecipitated with antiH4K8ac (Cell Signaling), anti-H3K9me3 (Cell Signaling).

\section{Bioinformatics analysis}

Array analysis was performed using Matlab (The MathWorks Inc.). Signals were extracted using Agilent Feature Extraction, quantile normalized and log2trasformed. Paired and unpaired T-test were applied to evaluate significantly deregulated miRNAs. For signature selection we considered as significant $p$ values less than 0.01. A False Discovery Rate procedure for multiple comparisons was also included in the analysis. Hierarchical Clustering and Principal Component Analysis were used to evaluate the efficacy of the selected signature.

Target prediction was assessed by using several prediction software included in the web server tool MirWalk2.0 (http://zmf.umm.uni-heidelberg.de/apps/zmf/mirwalk2/). Prediction was considered reliable if confirmed by at least three different software. Predicted targets were used for pathway analysis.

\section{qRT-PCR analysis}

10 ng of RNA was reverse-transcribed using the TaqMan microRNA Reverse Transcription Kit (Applied Biosystem) and Real time-PCR of miR expression was carried out using ABI Prism 7000 Sequence Detection System (Applied Biosystems). The PCR Reactions were initiated with a 10 min incubation at $95^{\circ} \mathrm{C}$ followed by 40 cycles of $95^{\circ} \mathrm{C}$ for $15 \mathrm{~s}$ and $60^{\circ} \mathrm{C}$ for $60 \mathrm{~s}$. RTq-PCR quantification of miRNA expression was performed using TaqMan MicroRNA ${ }^{\circ}$ Assays (Applied Biosystems) according to the manufacturer's protocol. RNU48 was used as endogenous control to normalize microRNA expression. All reactions were performed in duplicate.

\section{Transfection}

For mature miR-195-5p or miR-107 expression, we used Pre-miRNA Precursor-Negative Control (Ambion) and Pre-miRNA195-5p (Ambion) or Pre-miRNA107 at final concentration of 5nM. For miR-195-5p and miR-107 depletion we used miRCURY LNA microRNA inhibitor control (Exiqon) and hsa-miR-195-5p miRCURY LNA (Exiqon) or hsa-miR-107 miRCURY LNA (Exiqon) at final concentration of $10 \mathrm{nM}$. U87MG cells were transfected using Lipofectamine RNAiMAX (Invitrogen) according to the manufacturer's instructions. For miRNAs depletion experiments, after $48 \mathrm{~h}$ of transfection cells were treated with IC20 BRV or IC20 LCM for $48 \mathrm{~h}$.

\section{Immunoblotting analysis}

Cells were lysed in buffer consisting of $50 \mathrm{mM}$ Tris- $\mathrm{HCl}$ $\mathrm{pH} 8$, with 1\% NP-40 (Igepal AC-630) $150 \mathrm{mM} \mathrm{NaCl}$, $5 \mathrm{mM}$ EDTA and fresh protease inhibitors. Protein concentrations were determined by colorimetric assay (Bio-Rad). Western blotting was performed using the following primary antibodies: mouse monoclonal anti-Tubulin 
(Santa Cruz Biotechnology), mouse monoclonal antiGapdh (Santa Cruz Biotechnology), rabbit polyclonal anti-p21 (Santa Cruz Biotechnology), rabbit polyclonal anti-Cyclin A (Santa Cruz Biotechnology), mouse monoclonal anti-Cyclin E (Santa Cruz Biotechnology), rabbit monoclonal anti-EGFR (Cell Signaling Tecnology, C74B9), rabbit polyclonal anti-N-Cadherin (Abcam). Secondary antibodies used were goat anti-mouse and goat anti-rabbit conjugated to horseradish peroxidase (Santa Cruz Biotechnology).

\section{Cell proliferation assay}

U87MG cells $(6 \times 104)$ were transfected in triplicated as indicated. Cells were collected and counted at $0-24-48-$ $72 \mathrm{~h}$ after transfection.

\section{Migration assay}

Migration was measured using a 24-well plate with a non-coated 8-mm pore size filter in the insert chamber (BD Falcon). Cells were transfected with Pre-miRNA Precursor-Negative Control or the Pre-miRNA107, or the Pre-miRNA195-5p (Ambion), or treated with BRV or LCM at IC20. After $48 \mathrm{~h}$ from transfection or treatments, cells were resuspended in DMEM medium without FBS and seeded into the insert chamber. Cells were allowed to migrate for $12 \mathrm{~h}$ into the bottom chamber containing $0.7 \mathrm{ml}$ DMEM medium containing 10\% FBS in a humidified incubator at $37{ }^{\circ} \mathrm{C}$ in $5 \% \mathrm{CO} 2$. Migrated cells that had attached to the outside of the filter were visualized by staining with DAPI and counted.

\section{Statistical analysis}

Statistical analyses were performed by Pearson correlation coefficient for cytotoxicity assay and by Student- $t$ test for apoptosis, molecular analysis and cell cycle. Unless differently specified, level of significance was set at $p<0.05$.

\section{Results}

\section{Brivaracetam and lacosamide exert cytotoxic effect on} glioma cells and inhibit cell migration

In our experimental conditions, BRV and LCM displayed a dose-dependent cytotoxic effect in all glioma cell lines tested (Fig. 1) while no cytotoxic effect was detected in normal human fibroblasts (Additional file 1: Figure S1). A more detailed analysis in U87 revealed that the IC20 (i.e. $20 \%$ growth inhibiting concentration) were $400 \mu \mathrm{M}$ for BRV and $300 \mu \mathrm{M}$ for LCM. In T98G and SW1783 cell lines, IC20 were respectively $200 \mu \mathrm{M}$ and $821 \mu \mathrm{M}$ for BRV and $1178 \mu \mathrm{M}$ and $625 \mu \mathrm{M}$ for LCM.

No statistically significant effect of BRV or LCM was observed on apoptosis in U87MG. Even if a trend to increased apoptosis was observed $72 \mathrm{~h}$ after treatment with both drugs, this affects less than $4 \%$ of the cells
(Additional file 2: Figure S2a). Similarly, HUVECs did not display a statistically significant increase in apoptosis after in vitro exposure to the two drugs (Additional file 2: Figure S2b).

Upon BRV or LCM treatments (IC20) in T98G cells, we observed a specific arrest in G1 phase of cell cycle (Fig. 2a and Additional file 3: Figure S3 a-b). This effect on cell cycle was also confirmed by an increase in p21 protein expression levels, by a reduction in cyclin A protein levels and a concomitant accumulation of cyclin $\mathrm{E}$ protein observed in T98G, U87MG and SW1783 glioma cells upon BRV or LCM (IC20) treatments (Fig. 2b-d). To dipper investigate BRV and LCM effect on cell cycle, we also checked p53 protein levels upon BRV or LCM (IC20) observing an increase in its levels mainly in U87MG cells (p53 wild-type) and less evident in T98G and SW1783 cells (mutant p53) (Fig. 2b-d). This result suggested a specific effect of BRV and LCM on p53 protein depending on its mutational status.

Moreover, both treatments significantly impinged on U87MG cells migration (Fig. 2e). This effect was also confirmed by the reduction in N-cadherin and EGFR proteins expression levels in the same experimental conditions (Fig. 2f).

Brivaracetam and lacosamide treatments do not impinge on multidrug resistance proteins expression

Since regulation of multidrug resistance proteins expression might directly increase chemoresistance of glioma cells or decrease the permeability of the blood brain barrier to chemotherapic drugs, the expression of multidrug resistance proteins MRP1, MRP3, MRP5, PgP and GST $\pi$ was evaluated on U87MG cells and HUVEC after exposure to BRV and LCM IC20. No statistically significant differences in protein expression were observed after drug treatments compared to untreated cells (Additional file 4: Figure S4).

\section{Brivaracetam and lacosamide treatments modulate miRNAs expression in glioma cells}

To investigate if cytotoxic effect of BRV and LCM was mediated by miRNAs, we performed a miRNAs expression profile. We profiled the expression of 2000 human miRNAs in U87MG treated with BRV or LCM IC20 at the indicated time points (Fig. 3a-b). This kind of analysis identified a signature of 43 and 30 modulated miRNAs in U87MG treated with BRV and LCM respectively, 20 of which were shared by the two treatments (Fig. 3a-b, Additional file 5: Figure S5 and Additional file 6: Figure S6).

By performing pathway analysis of the putative target of both miRNAs signatures, we observed an almost complete overlapping between BRV and LCM treatments, possibly 


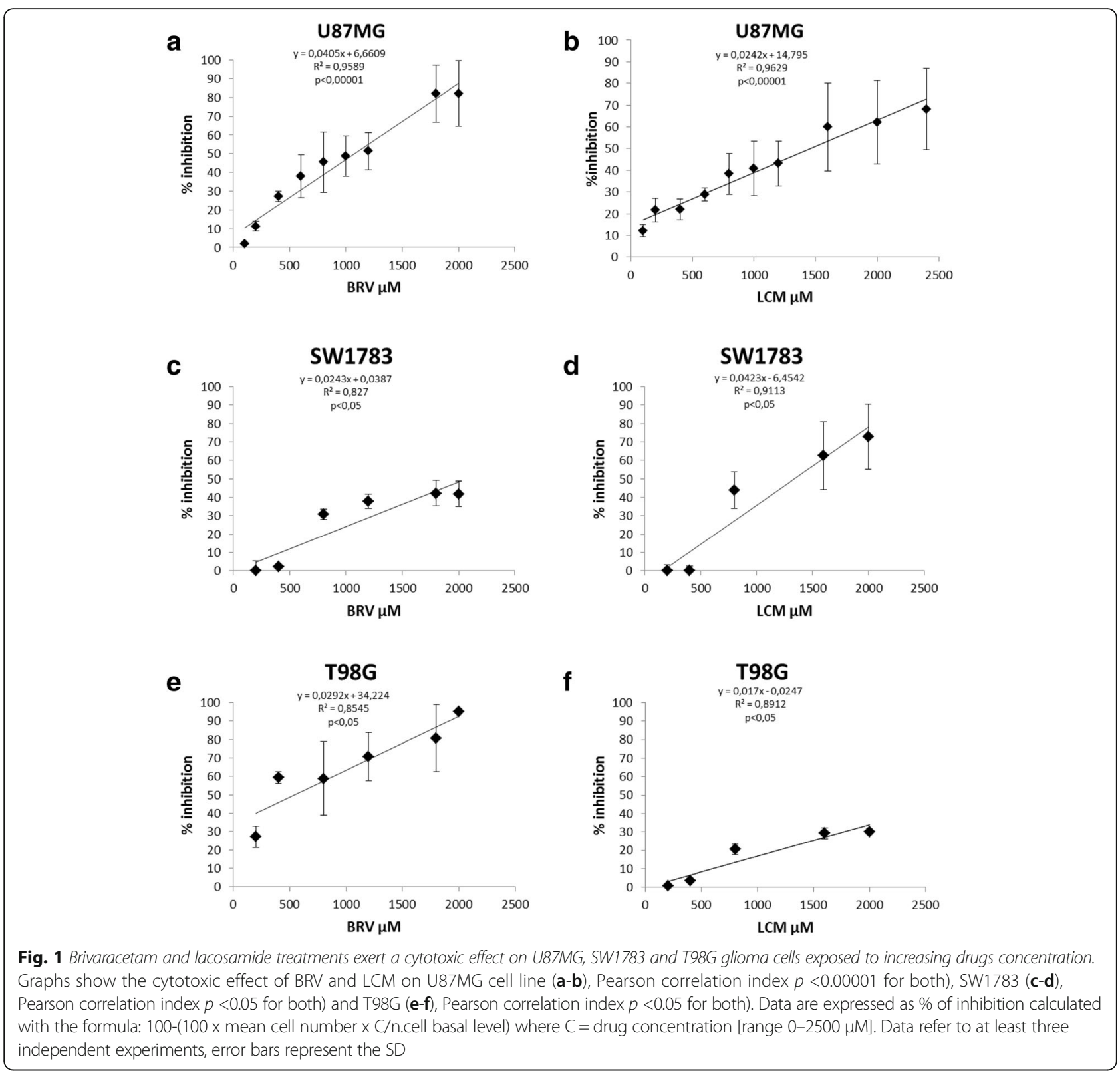

indicating a similar mechanism of action of the two AEDs (Table 1).

We focused our attention on two miRNAs whose expression was induced by both treatments compared to the control (untreated cells) and whose role in glioma tumorigenesis was partly characterized: miR-107 and miR-195-5p (Fig. 3c) [26-31]. To confirm these results, we analysed the expression levels of miR-107 and miR-195-5p by qRT-PCR (Fig. 3d). These analyses confirmed the up-regulation of both miRNAs upon BRV and LCM IC20 treatments at the indicated time points. Although at a minor extent, the increase in miR-195-5p expression was detected also in SW1783 and in T98G cell line upon treatments with both drugs, while in the latter cell line miR-107 was not inducible by LCM (Fig. 3e-f).

Brivaracetam and lacosamide treatments induce miR-107 and miR-195-5p expression in glioma cells by determining epigenetic modification on their regulatory regions

To evaluate if BRV- and LCM-mediated modulation of miR-107 and miR-195-5p expression occurred at the transcriptional level, we analysed the levels of their precursors (pre-miRNAs) upon BRV and LCM treatments in U87MG cells. As shown in Fig. 4a-b, both two AEDs increase pre-miRNAs levels, suggesting a transcriptional regulation of miR-107 and miR-195-5p. 
a

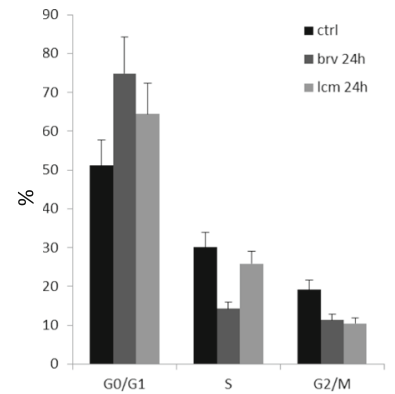

d

SW1783

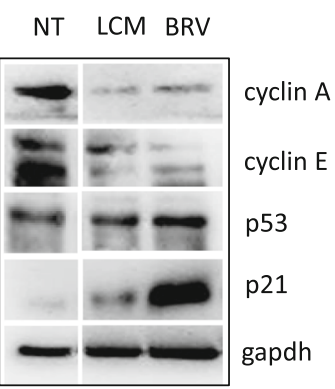

b
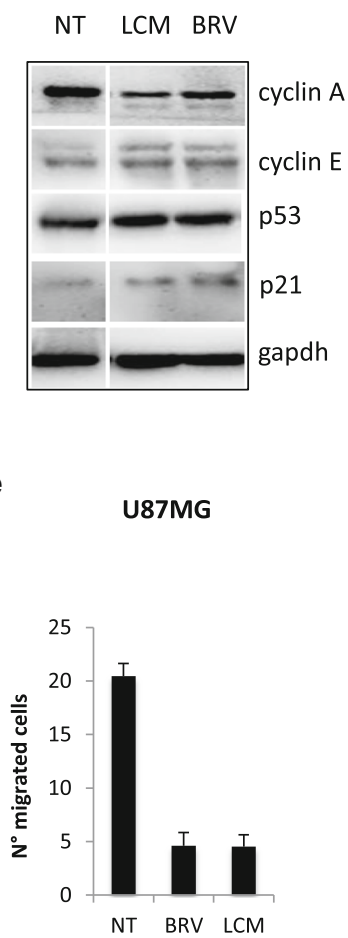

C

U87MG

NT BRV LCM

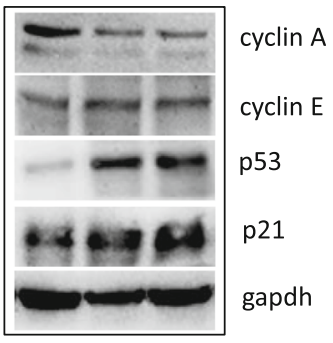

U87MG

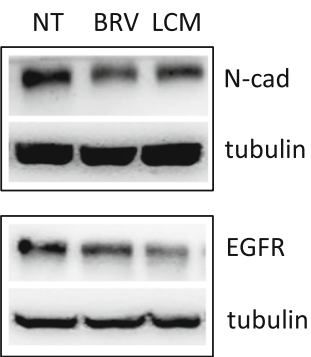

Fig. 2 Brivaracetam and lacosamide treatments exerted an anti-proliferative effect and a reduction in migration ability of glioma cells. a Distribution of T98G cells in the different phases of the cell cycle upon $24 \mathrm{~h} \mathrm{BRV}$ or LCM treatments (IC20). Data are expressed as percentage of cell in a specific phase (GO/G1, S, G2/M) and refers to at least four independent experiments. Statistical evaluation was performed by the student's t-test. Histogram bars represent mean \pm standard deviation of at least three independent replicates. b-c-d Western-blot analysis of cyclin A, cyclin E, p53 and p21 upon treatments with BRV or LCM at IC20 in T98G a U87MG b SW1783 c cells. e Transwell migration assay in U87MG cells upon BRV or LCM treatments. (IC20). $\mathbf{f}$ Western-blot analysis of EGFR and N-cadherin proteins expression levels in U87MG cells upon BRV or LCM treatments (IC20)

To deeper investigate the molecular mechanism involved in this regulation, we evaluate the ability of BRV and LCM to induce epigenetic modifications on the regulatory regions of $\mathrm{miR}-107$ and $\mathrm{miR}-195-5 \mathrm{p}$ genes. In particular, for miR-107, which is localized in the third intron of PANK1 gene, by analysing two regions of PANK1 promoter, we didn't observed a significant modification in the acetylation status of histone $\mathrm{H} 4$, that resulted highly acetylated in the control cells (untreated cells), while we observed a reduction in the methylation status of histone $\mathrm{H} 3$ in (Fig. 4c-e). Similar results were obtained for miR-195-5p, localized in the first intron of MIR495HG gene, by analysing two regions of MIR495HG promoter (Fig. 4f-h).

Altogether these findings indicated a BRV- and LCMmediated chromatin remodeling effect on miR-107 and miR-195 genes, in particular a reduction in the methylation status, determining miRNAs expression.

\section{MiR-195-5p inhibits U87MG cells proliferation}

To test if the cytotoxic effect of BRV and LCM was in part mediated by the induction of miR-195-5p or
miR-107, we ectopically expressed miR-195-5p or miR107 in U87MG and we evaluated cell proliferation rate. MiR-195-5p expression determined a significant reduction in U87MG growth and viability (Fig. 5a-b). On the other hand, miR-107 overexpression didn't determine any change (Additional file 7: Figure S7a-b).

Cell cycle analysis was also performed in U87MG cells ectopically expressing miR-195-5p (Fig. 5c and Additional file 7: Figure S7c). Overexpression of miR195-5p induced a significant increase in the percentage of cells in G0/G1 phase of the cell cycle after $48 \mathrm{~h}$ from transfection and a concomitant decrease in G2/M (Fig. 5c and Additional file 7: Figure S7c). These results are in line with the observed increase in p21 and cyclin E protein levels and decrease in cyclin A levels upon miR-195-5p over-expression or upon treatment with BRV or LCM (Figs. $5 \mathrm{~d}$ and $2 \mathrm{~b}-\mathrm{d}$ ). The anti-proliferative effect exerted by miR-195-5p was confirmed by cell morphology which presented the typical features of growth-arrested cells (Additional file 7: Figure S7d).

On the contrary, mir-195-5p depletion had no effect on U87MG cells viability and did not determine any 


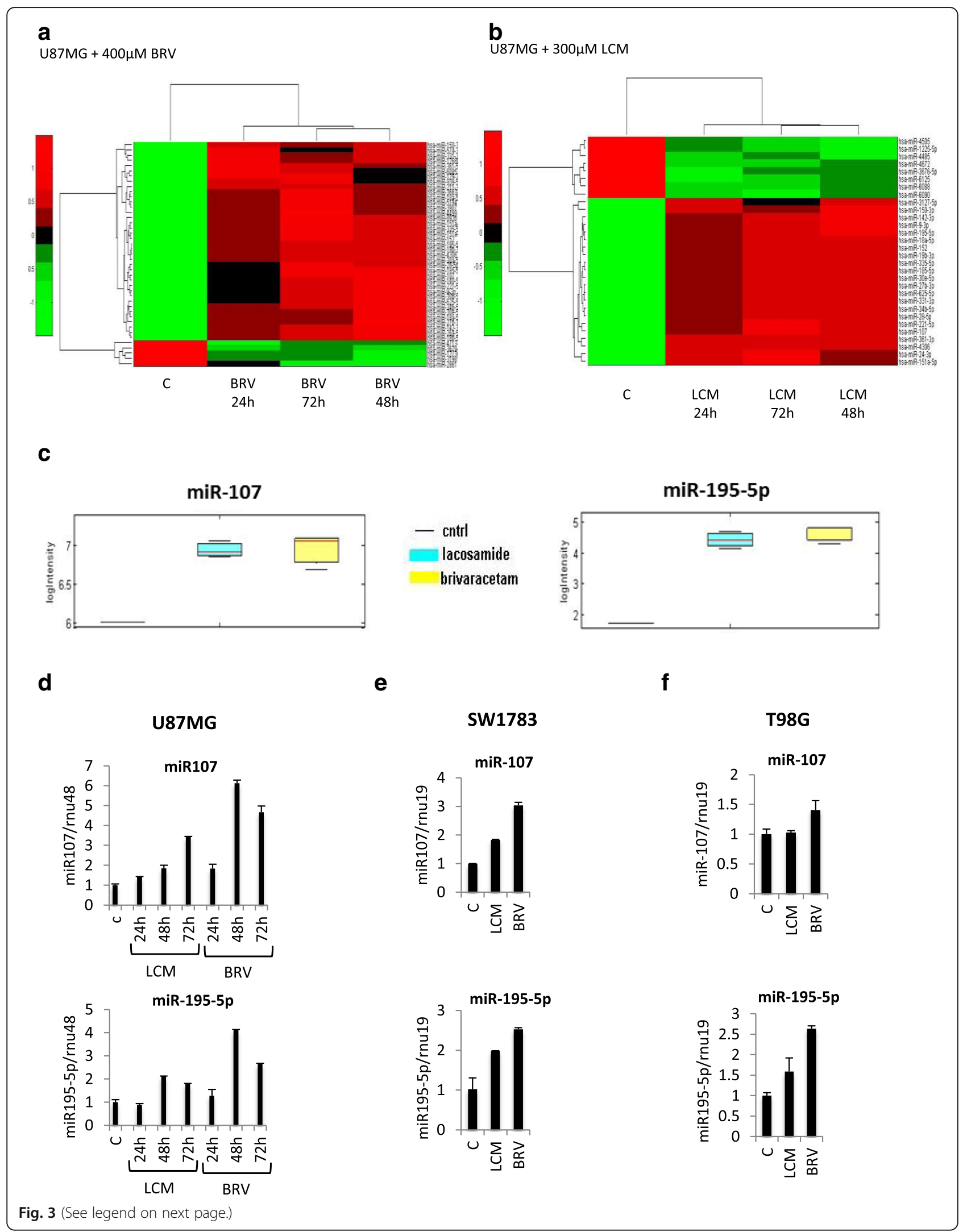


(See figure on previous page.)

Fig. 3 Brivaracetam and lacosamide treatments modulate microRNAs expression in U87MG glioma cells. a Heat map of the identified signature of 37 microRNAs differentially expressed in U87MG cells treated with BRV at IC20 at the indicated time points. $\mathbf{b}$ Heat map of the identified signature of 30 microRNAs differentially expressed in U87MG cells treated with LCM at IC20 at the indicated time points. c Supervised statistical test of the significance level of the difference between signal distributions of miR-107 and miR-195-5p in U87MG cells treated with LCM or BRV versus the control (untreated cells). d-e-f qRT-PCR validation of miR-107 and miR-195-5p in U87MG, SW1783 and T98G upon IC20 LCM or BRV treatments at the indicated time points

change in cyclin A, cyclin E and p21 protein levels (Fig. 5e-f Additional file 7: Figure S7e). Moreover, as shown in Fig. 5g-h, mir-195-5p silencing in U87MG cells abolished the anti-proliferative effects of BRV or LCM treatments. In particular miR-195-5p depletion blocked the BRV- or LCM- induced arrest in G1 phase of cell cycle and prevented cyclin $\mathrm{A}$ protein reduction and accumulation of cyclin E and p21 proteins (Fig. 5g-h).

\section{MiR-107 promotes U87MG cells migration}

BRV and LCM treatments in U87MG also determined a significant reduction in cell migration (Fig. 2e). We evaluated if this effect was mediated by miR-195-5p or miR107 modulation. MiR-107 significantly impinged on cell

Table 1 Predicted pathwhays targeted by miRNAs modulated by Brivaracetam and Lacosamide

\begin{tabular}{|c|c|c|c|}
\hline KEGG pathway & $p$-value & \#genes & \#miRNAs \\
\hline \multicolumn{4}{|l|}{ BRIVARACETAM } \\
\hline Proteoglycans in cancer & 6.85E-09 & 130 & 35 \\
\hline Fatty acid biosynthesis & 7.19E-08 & 8 & 11 \\
\hline TGF-beta signaling pathway & $1.74 \mathrm{E}-07$ & 55 & 32 \\
\hline AMPK signaling pathway & $5.90 \mathrm{E}-07$ & 84 & 36 \\
\hline Hippo signaling pathway & $1.68 \mathrm{E}-06$ & 93 & 33 \\
\hline Glutamatergic synapse & 7.59E-06 & 72 & 35 \\
\hline Long-term depression & $1.70 \mathrm{E}-05$ & 42 & 32 \\
\hline $\begin{array}{l}\text { Adrenergic signaling in } \\
\text { cardiomyocytes }\end{array}$ & $1.70 \mathrm{E}-05$ & 92 & 37 \\
\hline Pathways in cancer & $1.78 \mathrm{E}-05$ & 219 & 38 \\
\hline Axon guidance & $3.75 \mathrm{E}-05$ & 79 & 34 \\
\hline \multicolumn{4}{|l|}{ LACOSAMIDE } \\
\hline Proteoglycans in cancer & $5.142 \mathrm{E}-11$ & 120 & 22 \\
\hline Fatty acid biosynthesis & $5.755 \mathrm{E}-10$ & 8 & 10 \\
\hline Hippo signaling pathway & $5.755 E-10$ & 92 & 24 \\
\hline Axon guidance & $2.903 \mathrm{E}-07$ & 77 & 20 \\
\hline Long-term depression & 4.614E-07 & 43 & 21 \\
\hline TGF-beta signaling pathway & $1.372 \mathrm{E}-06$ & 49 & 20 \\
\hline Fatty acid metabolism & $1.636 \mathrm{E}-06$ & 23 & 17 \\
\hline $\begin{array}{l}\text { Adrenergic signaling in } \\
\text { cardiomyocytes }\end{array}$ & $1.771 \mathrm{E}-06$ & 86 & 24 \\
\hline $\begin{array}{l}\text { Signaling pathways regulating } \\
\text { pluripotency of stem cells }\end{array}$ & $3.548 \mathrm{E}-06$ & 82 & 22 \\
\hline Glutamatergic synapse & $3.548 \mathrm{E}-06$ & 66 & 23 \\
\hline
\end{tabular}

migration, while miR-195-5p had no effect (Fig. 6a and Additional file 8: Figure S8a). The inhibitory effect on cell migration was also verifiable in the levels of EGFR and $\mathrm{N}$-Cadherin proteins that were strongly reduced by miR-107 over-expressing cells (Fig. 6b) or by BRV and LCM treatments (Fig. 2c). On the contrary, miR-107 depletion improve migratory ability of U87MG cells as indicated by the increase of EGFR and N-cadherin protein levels (Fig. 6c). Moreover, miR-107 depletion prevented BRV- and LCM-mediated inhibition of cell migration (Fig. 6e and Additional file 8: Figure S8b).

\section{Discussion}

Epilepsy is a frequent complication in patients with brain tumors, therefore AED are widely used for seizure control in addition to surgery, chemotherapy and radiotherapy. For this reason a possible intrinsic antineoplastic effect of these drugs would be useful. In addition to the anticonvulsant mechanism, some AED have been previously described to exert a cytotoxic effect that, added to the effects of conventional chemo-radiotherapy, possibly impacts also on the survival of these patients $[10,16,17]$. Our results show that BRV and LCM in vitro exert a dose-dependent cytotoxic effect on various glioma cell lines and this effect was concomitant with the modulation of a number of miRNAs.

Although we could not completely clarify the mechanism of action, our data suggest that, even at low doses (IC20), the two drugs exert a role in blocking cell cycle progression of glioma cells possibly trough up-regulation of miR-195$5 p$. Indeed, in our experimental conditions, these two drugs seem to impinge on G1/S phase transition of cell cycle, as demonstrated by reduction in cyclin A protein levels and increase in p21 and cyclin E protein levels after treatments.

The tumor-suppressor activity of miR-195-5p in glioma cells and other tumor models has been previously characterized [29-31]. Zhang et al. reported that overexpression of miR-195-5p was able to induce the arrest of cell cycle progression in G1/S transition in U87 [31] and similar results have been described by Hui et al. in other cellular models of human glioma [29]. An increase in p21 expression has been also previously reported to be associated to cell cycle arrest in lung, colorectal, thyroid and kidney cell lines [32-34]. In agreement with these data, our results confirm the role of miR-195-5p in the suppression of cell proliferation in human glioma cells. 

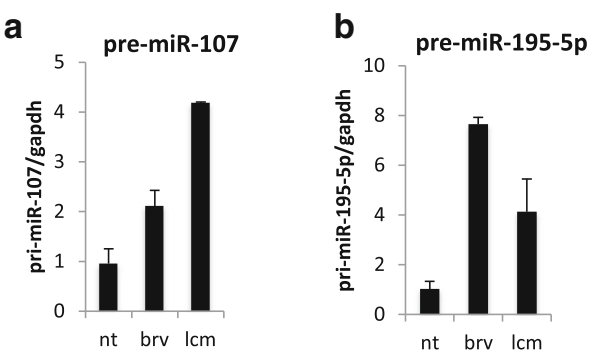

C
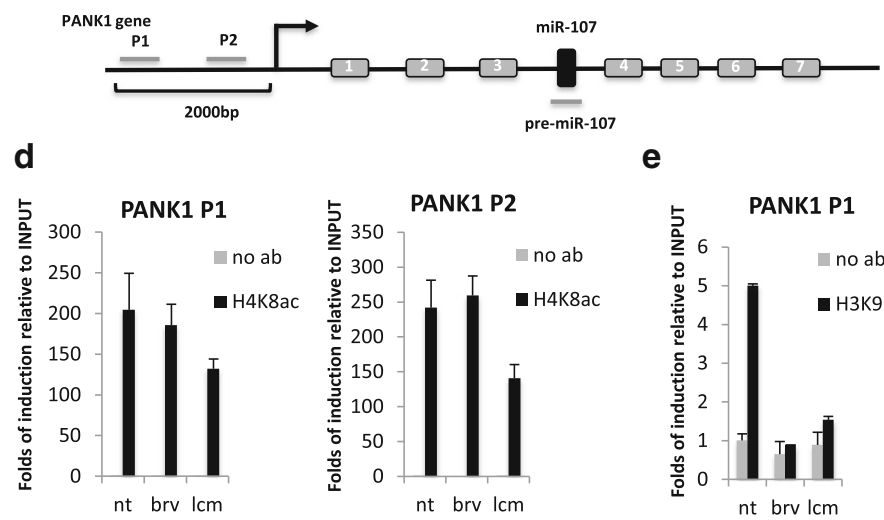

e

\section{f}

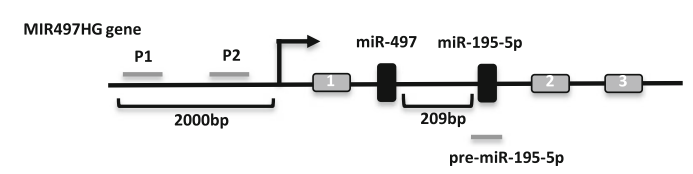

g

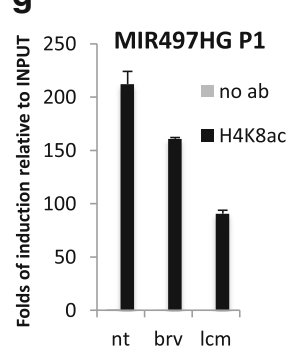

h

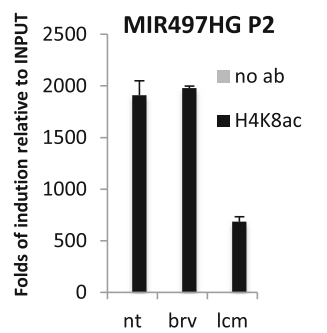

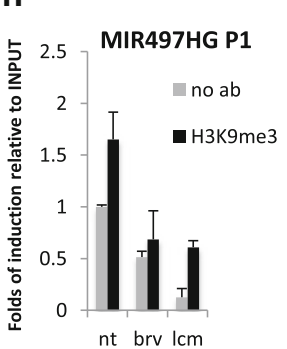

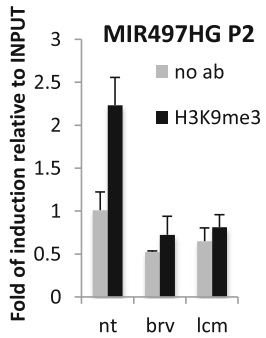

Fig. 4 Brivaracetam and lacosamide treatments induce epigenetic modification on miR-107 and miR-195 regulatory regions. a-b qRT-PCR of miR-107 $\mathbf{a}$ and miR-195-5p b precursors (pre-miRNAs) in U87MG cells upon IC20 LCM or BRV treatments. c Schematic representation of miR-107 gene locus: two different regions of host gene (PANK1) promoter have been analyzed in ChIP assays (P1 and P2). d-e ChIP analysis of acetylated histone $\mathrm{H} 4$ and methylated histone H3 occupancy on miR-107 regulatory regions in U87MG cells upon treatment with BRV or LCM at IC20. f Schematic representation of miR-195-5p gene locus: two different regions of host gene (MIR497HG) promoter have been analyzed in ChIP assays (P1 and P2). g-h ChIP analysis of acetylated histone H4 and methylated histone H3 occupancy on miR-195-5p regulatory regions in U87MG cells upon treatment with BRV or LCM at IC20

Other AED have been previously reported to interfere with cell cycle in cancer cells [35-37]. Bobustuc and colleagues reported that levetiracetam inhibited in vitro human glioma cell proliferation through p53-mediated MGMT inhibition, thus increasing glioma cell sensitivity to temozolomide [10]. Moreover, in a recent clinical study, Kim and colleagues showed that patients with glioblastoma receiving temozolomide-based chemotherapy and levetiracetam for seizure control, experienced a significant survival benefits [16]. Our in vitro data suggest that some characteristics of the parent drug levetiracetam might be shared by BRV, however further study is needed to verify the effect of BRV on MGMT expression.

Moreover, our results show that BRV and LEV may modulate other miRNAs. Over-expression of miR-107 has been also demonstrated to inhibit glioma cell growth increasing apoptosis [28] and to inhibit cell 
a

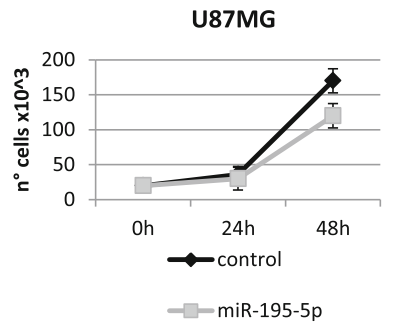

b

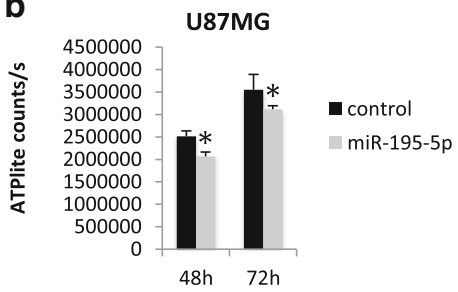

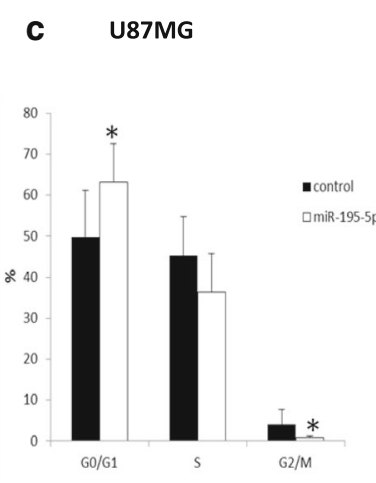

f

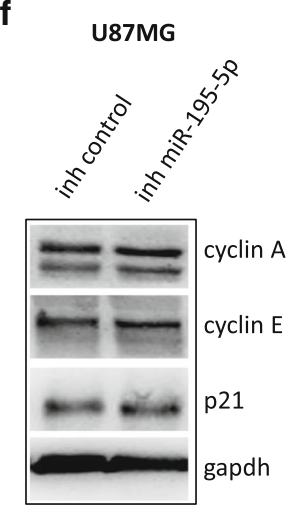

d

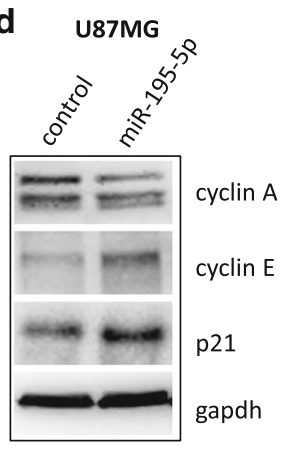

g

U87MG

e

U87MG

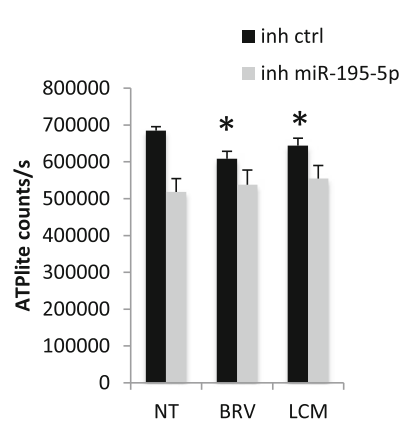

h

U87MG
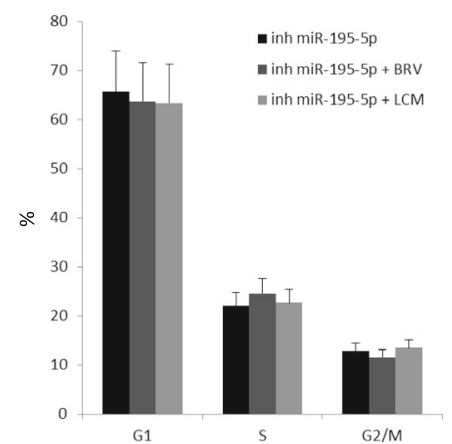

inh miR-195-5p

NT BRV LCM

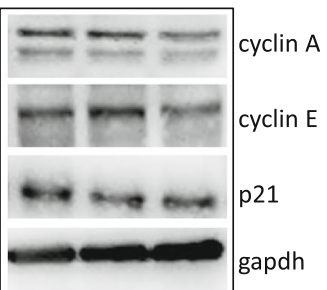

Fig. 5 Brivaracetam and lacosamide treatments exert their anti-proliferative effect in part trough miR-195-5p. a-b Proliferation assay (a) and viability assay b in U87MG cells transfected with miR-195-5p mimic or control. Cells were collected and counted at the indicated time points. c Distribution of U87MG cells in the different phases of the cell cycle. Data are expressed as percentage of cell in a specific phase (G0/G1, S, G2/M) and refers to at least four independent experiments. Statistical evaluation was performed by the student's t-test. Histogram bars represent mean \pm standard deviation of at least three independent replicates. $\mathbf{d}$ Western-blot analysis of cyclin A, cyclin E and p21 protein expression levels in U87MG cells upon miR-195-5p over-expression. e Viability assay in U87MG cells transfected with miR-195-5p inhibitor or control and treated with BRV or LCM at IC20. f Western-blot analysis of cyclin A, cyclin E and p21 protein expression levels in U87MG cells upon miR-195-5p depletion with miR-195-5p inhibitor (inh miR-195-5p). g Distribution of U87MG cells in the different phases of the cell cycle upon miR-195 depletion and treatments wit BRV or LCM at IC20. Data are expressed as percentage of cell in a specific phase (G0/G1, S, G2/M) and refers to at least four independent experiments. Statistical evaluation was performed by the student's t-test. Histogram bars represent mean \pm standard deviation of at least three independent replicates. $\mathbf{h}$ Western-blot analysis of cyclin A, cyclin E and p21 protein expression levels in U87MG cells upon miR-195-5p depletion with miR-195-5p inhibitor (inh miR-195-5p) and treatments wit BRV or LCM at IC20. $\left(^{*}=p\right.$ val $\left.<0.05\right)$

migration/invasion [26, 27]. In our experimental conditions after treatment with LCM or BRV, the percentage of apoptotic cells was barely detectable therefore too low to be responsible of the observed cytotoxicity. On the other hand, our data confirm that overexpression of miR-107 reduces the ability of glioma cells to migrate in an in vitro assay.
Exposure to BRV and LCM seems to have no impact on chemoresistance. Indeed ATP-dependent drug efflux transporters or multidrug resistance proteins have been localized in different tissues including the blood brain barrier and they affect absorption, distribution and excretion of different drugs [38-41]. Although preliminary, our results on endothelial and glioma cells suggest 


\section{a}

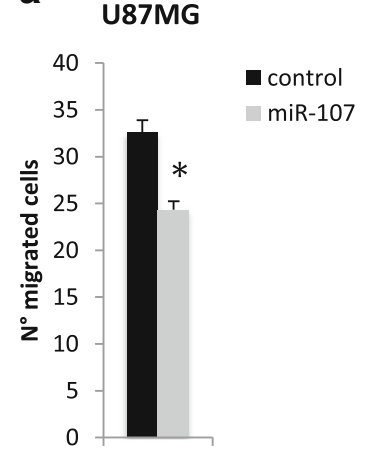

b U87MG

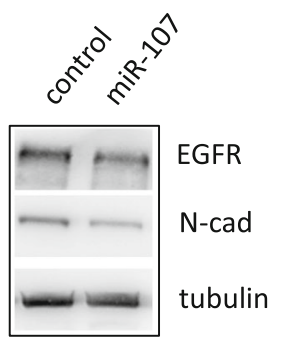

C

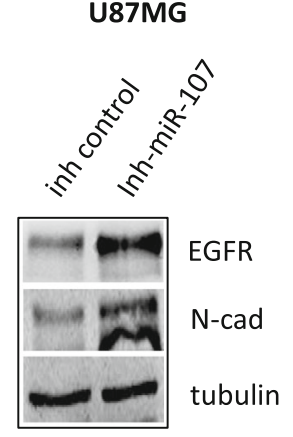

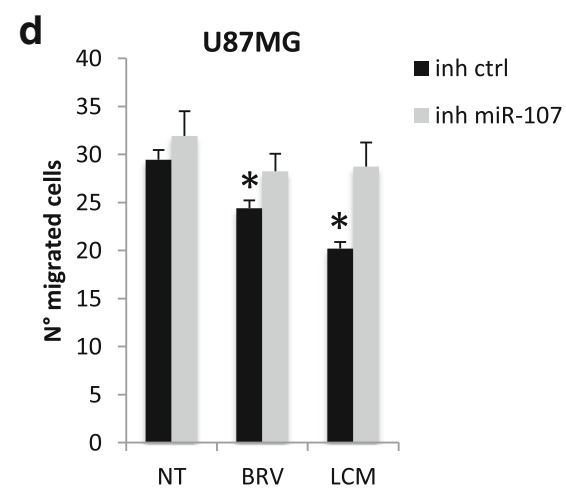

Fig. 6 Brivaracetam and lacosamide treatments inhibit glioma cells migration in part trough miR-107. a Transwell migration assay in U87MG cells upon miR-107 exogenous expression $\left(^{*}=p v a l<0.05\right)$. b-c Western-blot analysis of EGFR and N-cadherin proteins expression levels in U87MG cells upon miR-107 over-expression with miR-107 mimic (miR-107) (b) or depletion with miR-107 inhibitor (inh miR-107) (c). d Transwell migration assay in U87MG cells upon miR-107 depletion with miR-107 inhibitor (inh miR-107)

that the administration of these two drugs would not significantly modify glioma cells chemoresistance or the availability of chemotherapic drugs at the blood/tumor interface.

The meaning of the decreased expression of $\mathrm{N}$-cadherin and EGFR following miR-107 overexpression deserves further investigation.

In U87 cells the decrease in N-cadherin protein levels correlates with a reduced migratory ability of the cells upon ectopic expression of miR-107 as upon treatment with BRV or LCM. The role of N-cadherin in the migration of mesenchymal cells is not completely defined. Recently, Guo et al. observed a reduction in the expression of mesenchymal related protein, such as $\mathrm{N}$-cadherin, that determined a mesenchymal-to-epithelial transition of U87 and a consequent inhibition of cell migration [42]. This is in line with our observations.

Amplified expression of EGFR and of its mutated variant v3, has been extensively studied as a possible target for anti-tumor therapy, although clinical trials focused on this treatment approach have so far yielded unsatisfactory results [43]. A positive correlation between EGFR expression and migration ability has been previously reported in glioma cell lines and in neuronal stem cells therefore our results would further support the role of EGFR in cell motility $[44,45]$.

\section{Conclusions}

Even if at concentration higher than those recommended for epilepsy control, our study provides evidence for possible effects of two novel anti-epileptic drugs, both devoid of enzyme-inducing activity, on glioblastoma cell proliferation/migration, at least partly mediated by enhanced expression of 2 miRNAs.

Further studies are needed to better delineate the multiple biological effects of these drugs in glioma and their possible impact on clinical management and outcome in brain tumor-related epilepsy.

\section{Additional files}

Additional file 1: Figure S1. Brivaracetam and lacosamide treatments displayed no cytotoxic effect on normal human fibroblast exposed to increasing drugs concentration. Data refers to at least three independent experiments and are expressed as cell number \pm SD. (PPTX $106 \mathrm{~kb}$ ) 
Additional file 2: Figure S2. a-b) Apoptosis analysis studied on the cel line U87MG (a) and on HUVECs (b) after treatment with BRV (IC20, grey histogram) and LCM (IC20, empty histogram) at different time points 24, 48 and $72 \mathrm{~h}$. Data are expressed as difference in the \% of apoptotic cells between treated and untreated cells ( $\Delta$ apoptotic inhibition). Data refer to at least three independent experiments, error bars represent the SD. (PPTX $72 \mathrm{~kb}$ )

Additional file 3: Figure S3. Brivaracetam and lacosamide treatments induces accumulation of cells in G0/G1. a-b) Distribution of T98G cells in the different phases of the cell cycle upon $48 \mathrm{~h}$ (a) or $72 \mathrm{~h}$ (b) BRV or LCM treatments (IC20). Data are expressed as percentage of cell in a specific phase $(G 0 / G 1, S, G 2 / M)$ and refers to at least four independent experiments. Statistical evaluation was performed by the student's t-test. Histogram bars represent mean \pm standard deviation of at least three independent replicates. (PPTX $65 \mathrm{~kb}$ )

Additional file 4: Figure S4. Brivaracetam and lacosamide treatments have no impact on chemoresistence induction. Drug resistance molecules expression and modulation in U87MG cell line as detected by flow cytometry. In vitro cultured cells were treated with an IC20 concentration of BRV (grey histogram) or LCM (empty histogram) for $72 \mathrm{~h}$, harvested and labelled with the specific antibodies (see methods). Data are expressed as fold increase/decrease (means \pm SD) of treated cells compared to basal expression (averages of mean fluorescence intensity of treated cells/averages of mean fluorescence intensity of untreated cells). (PPTX $196 \mathrm{~kb}$ )

Additional file 5: Figure S5. miRNAs modulated in U87MG cells upon LCM treatment. Differentiating miRNAs are listed with their $p$-values obtained by paired t-test ( $p$ val). In the table are also indicated false discovery rate values (FDR), and folds of deregulation expressed in logarithmic scale (log2 fold). (PPTX $63 \mathrm{~kb}$ )

Additional file 6: Figure S6. miRNAs modulated in U87MG cells upon BRV treatment. Differentiating miRNAs are listed with their $p$-values obtained by paired t-test (pval). In the table are also indicated false discovery rate values (FDR), and folds of deregulation expressed in logarithmic scale (log2 fold). (PPTX $64 \mathrm{~kb}$ )

Additional file 7: Figure S7. Ectopic expression of miR-195-5p induces accumulation of cells in G0/G1. a-b) Proliferation assay (a) and viability assay (b) in U87MG cells transfected with miR-107 mimic or control. Cells were collected and counted at the indicated time points. c) Typical experiment. U87MG were transfected with control (left) or with mimic miR-195-5p mimic (right) and cultured for the following $48 \mathrm{~h}$. Cell were then harvested, fixed in $80 \%$ ethanol, stained with PI and analysed by flow cytometry for DNA content (see methods). d) U87MG cells morphology upon miR-195-5p exogenous expression. e) qRT-PCR of miR-195-5p in U87MG cells depleted for miR-195-5p (inh miR-195-5p) and treated with BRV or LCM (IC20). (PPTX $3351 \mathrm{~kb}$ )

Additional file 8: Figure 58. a) Transwell migration assay in U87MG cells upon miR-195-5p exogenous expression. b) qRT-PCR of miR-107 in U87MG cells depleted for miR-107 (inh miR-107) and treated with BRV or LCM (IC20). (PPTX $44 \mathrm{~kb}$ )

\section{Abbreviations}

AED: Anti-epileptic drugs; BRV: Brivaracetam; EIAED: Enzyme-inducing anticonvulsant drugs; GBM: Glioblastoma; HUVECs: Human umbilical vein endothelial cells; LCM: Lacosamide; LEV: Levetiracetam; miRNA: microRNA MRPs: Multidrug resistance proteins; OS: Overall survival; PFS: Progression-free survival; TMZ: Temozolomide; VPA: Valproic acid

\section{Acknowledgments}

The authors wish to thank Dr. Chiara Calatozzolo and Dr. Fiona Zucchetti for their precious help in cytotoxicity tests and in discussing the results.

\section{Funding}

This study was partially supported by UCB Pharma grant $n$. RS235/12, AIRC grant n. 14455 and EPIGEN grant n. 13/05/R/42.

\section{Availability of data and materials}

All data generated or analyzed during this study are included in this published article. Raw and processed data are stored in the laboratory of the corresponding authors and are available upon request.

\section{Authors' contributions}

$A R, S D, A S a l, G B, M M, E C$ conceived and designed the experiments. AR, SD, $V G, C V$ performed most of the experiments. ASac performed bioinformatics analysis. AR, SD, ASal, GB, MM, EC contributed to the writing of the manuscript. All the authors read and approved the final version of this manuscript.

\section{Competing interests}

AR was partially supported by UCB Pharma (RS235/12). GB was partially supported by AIRC (14455) and EPIGEN (13/05/R/42). MM declare to have had an indirect financial relationship with UCB Pharma.

\section{Consent for publication}

Not applicable.

\section{Ethics approval and consent to participate}

Not applicable.

\section{Publisher's Note}

Springer Nature remains neutral with regard to jurisdictional claims in published maps and institutional affiliations.

\section{Author details}

'Laboratory of Clinical Pathology and Medical Genetics, Foundation IRCCS Neurological Institute C. Besta, Via Celoria, 11, 20133 Milan, Italy.

${ }^{2}$ Oncogenomic and Epigenetic Unit, Regina Elena National Cancer Institute, Via Elio Chianesi, 5300144 Rome, Italy. ${ }^{3}$ Neurologia- Stroke Unit, Manzoni Hospital, Via dell'Eremo 9/11, 23900 Lecco, Italy. ${ }^{4}$ Center for tumor-related epilepsy, Area of Supporting Care, Regina Elena National Cancer Institute, Via Elio Chianesi 53, 00144 Rome, Italy.

Received: 6 March 2017 Accepted: 29 May 2017

Published online: 06 June 2017

\section{References}

1. Blomqvist $P$, Lycke J, Strang $P$, Tornqvist H, Ekbom A. Brain tumours in Sweden 1996: care and costs. J Neurol Neurosurg Psychiatry. 2000;69(6):792-8.

2. Ekman M, Westphal M. Cost of brain tumour in Europe. Eur J Neurol. 2005; 12 Suppl 1:45-9.

3. Gustavsson A, Svensson M, Jacobi F, Allgulander C, Alonso J, Beghi E, Dodel R, Ekman M, Faravelli C, Fratiglioni L, Gannon B, Jones DH, Jennum P, Jordanova A, Jonsson L, Karampampa K, et al. Cost of disorders of the brain in Europe 2010. Eur Neuropsychopharmacol. 2011;21(10):718-79.

4. Latif $A Z$, Signorini $D$, Gregor A, Whittle IR. The costs of managing patients with malignant glioma at a neuro-oncology clinic. Br J Neurosurg. 1998. 12(2):118-22.

5. Maschio M, Sperati F, Dinapoli L, Vidiri A, Fabi A, Pace A, Pompili A, Carapella CM, Cantelmi T. Weight of epilepsy in brain tumor patients. J Neuro-Oncol. 2014;118(2):385-93.

6. Schiff D, Lee EQ, Nayak L, Norden AD, Reardon DA, Wen PY. Medical management of brain tumors and the sequelae of treatment. Neuro-Oncology. 2015;17(4):488-504.

7. Vecht CJ, van Breemen M. Optimizing therapy of seizures in patients with brain tumors. Neurology. 2006;67(12 Suppl 4):S10-3.

8. Patsalos PN, Perucca E. Clinically important drug interactions in epilepsy: interactions between antiepileptic drugs and other drugs. Lancet Neurol. 2003;2(8):473-81.

9. Weller M, Stupp R, Wick W. Epilepsy meets cancer: when, why, and what to do about it? Lancet Oncol. 2012;13(9):e375-82.

10. Bobustuc GC, Baker CH, Limaye A, Jenkins WD, Pearl G, Avgeropoulos NG, Konduri SD. Levetiracetam enhances p53-mediated MGMT inhibition and sensitizes glioblastoma cells to temozolomide. Neuro-Oncology. 2010;12(9): 917-27.

11. Jaeckle KA, Ballman K, Furth A, Buckner JC. Correlation of enzyme-inducing anticonvulsant use with outcome of patients with glioblastoma. Neurology. 2009;73(15):1207-13. 
12. Weller M, Gorlia T, Cairncross JG, van den Bent MJ, Mason W, Belanger K, Brandes AA, Bogdahn U, Macdonald DR, Forsyth P, Rossetti AO, Lacombe D, Mirimanoff RO, Vecht CJ, Stupp R. Prolonged survival with valproic acid use in the EORTC/NCIC temozolomide trial for glioblastoma. Neurology. 2011; 77(12):1156-64.

13. Berendsen S, Broekman M, Seute T, Snijders T, van Es C, de Vos F, Regli L, Robe P. Valproic acid for the treatment of malignant gliomas: review of the preclinical rationale and published clinical results. Expert Opin Investig Drugs. 2012;21(9):1391-415

14. Guthrie GD, Eljamel S. Impact of particular antiepileptic drugs on the survival of patients with glioblastoma multiforme. J Neurosurg. 2013;118(4):859-65.

15. Kerkhof M, Dielemans JC, van Breemen MS, Zwinkels H, Walchenbach $R$ Taphoorn MJ, Vecht CJ. Effect of valproic acid on seizure control and on survival in patients with glioblastoma multiforme. Neuro-Oncology. 2013; 15(7):961-7.

16. Kim YH, Kim T, Joo JD, Han JH, Kim YJ, Kim IA, Yun CH, Kim CY. Survival benefit of levetiracetam in patients treated with concomitant chemoradiotherapy and adjuvant chemotherapy with temozolomide for glioblastoma multiforme. Cancer. 2015;121(17):2926-32.

17. Eyal S, Yagen B, Sobol E, Altschuler Y, Shmuel M, Bialer M. The activity of antiepileptic drugs as histone deacetylase inhibitors. Epilepsia. 2004;45(7):737-44.

18. Kumar MS, Lu J, Mercer KL, Golub TR, Jacks T. Impaired microRNA processing enhances cellular transformation and tumorigenesis. Nat Genet. 2007;39(5):673-7

19. Mallardo M, Poltronieri P, D'Urso OF. Non-protein coding RNA biomarkers and differential expression in cancers: a review. J Exp Clin Cancer Res. 2008;27:19.

20. Gurtner A, Falcone E, Garibaldi F, Piaggio G. Dysregulation of microRNA biogenesis in cancer: the impact of mutant p53 on Drosha complex activity. J Exp Clin Cancer Res. 2016:35:45

21. You G, Yan W, Zhang W, Wang Y, Bao Z, Li S, Li S, Li G, Song Y, Kang C, Jiang T. Significance of miR-196b in tumor-related epilepsy of patients with gliomas. PLoS One. 2012;7(9):e46218.

22. Bang SR, Ambavade SD, Jagdale PG, Adkar PP, Waghmare AB, Ambavade PD. Lacosamide reduces HDAC levels in the brain and improves memory: Potential for treatment of Alzheimer's disease. Pharmacol Biochem Behav. 2015;134:65-9

23. May TW, Brandt C, Helmer R, Bien CG, Cawello W. Comparison of lacosamide concentrations in cerebrospinal fluid and serum in patients with epilepsy. Epilepsia. 2015;56(7):1134-40.

24. Nicolas JM, Hannestad J, Holden D, Kervyn S, Nabulsi N, Tytgat D, Huang Y, Chanteux H, Staelens L, Matagne A, Mathy FX, Mercier J, Stockis A, Carson $\mathrm{RE}$, Klitgaard H. Brivaracetam, a selective high-affinity synaptic vesicle protein 2A (SV2A) ligand with preclinical evidence of high brain permeability and fast onset of action. Epilepsia. 2016;57(2):201-9.

25. Jaffe EA, Nachman RL, Becker CG, Minick CR. Culture of human endothelial cells derived from umbilical veins. Identification by morphologic and immunologic criteria. J Clin Invest. 1973;52(11):2745-56.

26. Chen L, Chen XR, Chen FF, Liu Y, Li P, Zhang R, Yan K, Yi YJ, Xu ZM, Jiang XD. MicroRNA-107 inhibits U87 glioma stem cells growth and invasion. Cell Mol Neurobiol. 2013;33(5):651-7.

27. Chen L, Chen XR, Zhang R, Li P, Liu Y, Yan K, Jiang XD. MicroRNA-107 inhibits glioma cell migration and invasion by modulating Notch2 expression. J Neuro-Oncol. 2013;112(1):59-66.

28. He J, Zhang W, Zhou Q, Zhao T, Song Y, Chai L, Li Y. Low-expression of microRNA-107 inhibits cell apoptosis in glioma by upregulation of SALL4. Int J Biochem Cell Biol. 2013;45(9):1962-73.

29. Hui W, Yuntao L, Lun L, WenSheng L, ChaoFeng L, HaiYong H, Yueyang B. MicroRNA-195 inhibits the proliferation of human glioma cells by directly targeting cyclin D1 and cyclin E1. PLoS One. 2013;8(1):e54932.

30. Yang G, Wu D, Zhu J, Jiang O, Shi Q, Tian J, Weng Y. Upregulation of miR195 increases the sensitivity of breast cancer cells to Adriamycin treatment through inhibition of Raf-1. Oncol Rep. 2013;30(2):877-89.

31. Zhang QQ, Xu H, Huang MB, Ma LM, Huang QJ, Yao Q, Zhou H, Qu LH. MicroRNA-195 plays a tumor-suppressor role in human glioblastoma cells by targeting signaling pathways involved in cellular proliferation and invasion. Neuro-Oncology. 2012;14(3):278-87.

32. Ding $L$, Huang $Y$, Dai $M$, Zhao $X$, Du Q, Dong F, Wang L, Huo R, Zhang W, Xu X, Tong D. Transmissible gastroenteritis virus infection induces cell cycle arrest at $\mathrm{S}$ and G2/M phases via p53-dependent pathway. Virus Res. 2013; 178(2):241-51.
33. Gottifredi V, McKinney K, Poyurovsky MV, Prives C. Decreased p21 levels are required for efficient restart of DNA synthesis after $S$ phase block. J Biol Chem. 2004;279(7):5802-10.

34. Liu Z, Yang $L$, Teng $X$, Zhang $H$, Guan $H$. The involvement of CXCR7 in modulating the progression of papillary thyroid carcinoma. J Surg Res. 2014; 191(2):379-88.

35. Chen J, Ouyang Y, Cao L, Zhu W, Zhou Y, Zhou Y, Zhang H, Yang X, Mao L, Lin S, Lin J, Hu J, Yan G. Diazepam inhibits proliferation of human glioblastoma cells through triggering a G0/G1 cell cycle arrest. J Neurosurg Anesthesiol. 2013;25(3):285-91.

36. Kwiecinska P, Tauboll E, Gregoraszczuk EL. Effects of valproic acid and levetiracetam on viability and cell cycle regulatory genes expression in the OVCAR-3 cell line. Pharmacol Rep. 2012;64(1):157-65.

37. Takai N, Kawamata N, Gui D, Said JW, Miyakawa I, Koeffler HP. Human ovarian carcinoma cells: histone deacetylase inhibitors exhibit antiproliferative activity and potently induce apoptosis. Cancer. 2004; 101(12):2760-70.

38. Baltes S, Gastens AM, Fedrowitz M, Potschka H, Kaever V, Loscher W. Differences in the transport of the antiepileptic drugs phenytoin, levetiracetam and carbamazepine by human and mouse P-glycoprotein. Neuropharmacology. 2007;52(2):333-46.

39. Fromm MF. Importance of P-glycoprotein at blood-tissue barriers. Trends Pharmacol Sci. 2004;25(8):423-9.

40. Schinkel AH, Jonker JW. Mammalian drug efflux transporters of the ATP binding cassette (ABC) family: an overview. Adv Drug Deliv Rev. 2003;55(1):3-29.

41. Guo Z, Zhu J, Zhao L, Luo Q, Jin X. Expression and clinical significance of multidrug resistance proteins in brain tumors. J Exp Clin Cancer Res. 2010;29:122.

42. Guo G, Yao W, Zhang Q, Bo Y. Oleanolic acid suppresses migration and invasion of malignant glioma cells by inactivating MAPK/ERK signaling pathway. PLoS One. 2013;8(8):e72079.

43. Roth $\mathrm{P}$, Weller M. Challenges to targeting epidermal growth factor receptor in glioblastoma: escape mechanisms and combinatorial treatment strategies. Neuro Oncol. 2014;16 Suppl 8:viii14-19.

44. Boockvar JA, Kapitonov D, Kapoor G, Schouten J, Counelis GJ, Bogler O, Snyder EY, McIntosh TK, O'Rourke DM. Constitutive EGFR signaling confers a motile phenotype to neural stem cells. Mol Cell Neurosci. 2003;24(4):1116-30

45. Katakowski M, Zheng X, Jiang F, Rogers T, Szalad A, Chopp M. MiR-146b-5p suppresses EGFR expression and reduces in vitro migration and invasion of glioma. Cancer Investig. 2010;28(10):1024-30.

\section{Submit your next manuscript to BioMed Central and we will help you at every step:}

- We accept pre-submission inquiries

- Our selector tool helps you to find the most relevant journal

- We provide round the clock customer support

- Convenient online submission

- Thorough peer review

- Inclusion in PubMed and all major indexing services

- Maximum visibility for your research

Submit your manuscript at www biomedcentral.com/submit
C BioMed Central 